View Journal | View Issue

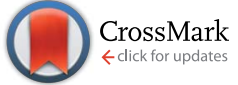

Cite this: Chem. Sci., 2016, 7, 2929

\title{
Reply to the 'Comment on "Theoretical studies on a carbonaceous molecular bearing: association thermodynamics and dual-mode rolling dynamics"' by E. M. Cabaleiro-Lago, J. Rodriguez-Otero and A. Gil, Chem. Sci., 2016, 7, DOI: 10.1039/C5SC04676A
}

\author{
Hiroyuki Isobe, ${ }^{\text {ab }}$ Kosuke Nakamura, ${ }^{\mathrm{b}}$ Shunpei Hitosugi, ${ }^{\mathrm{b}}$ Sota Sato, ${ }^{\text {ab }}$ \\ Hiroaki Tokoyama, ${ }^{c}$ Hideo Yamakado, ${ }^{c}$ Koichi Ohno ${ }^{\text {bd }}$ and Hirohiko Kono*b
}

Received 3rd February 2016

Accepted 8th February 2016

DOI: $10.1039 / \mathrm{c} 6 \mathrm{sc00550k}$

www.rsc.org/chemicalscience

We reply to the comments raised by Cabaleiro-Lago et al. on our article ( $\mathrm{H}$. Isobe et al., Chem. Sci., 2015, 6, 27462753). Here we appreciate the common views we share and welcome the clarity this gives, and we discuss and question some of their criticisms of the original piece and clarify our opinion in this area. The most important answer can be found in the equation, $\Delta G=\Delta H-T \Delta S$, derived from one of the best established fields in physics.

Reading the comments from Cabaleiro-Lago et al., ${ }^{1}$ we were delighted to learn that the first benchmark results for the thermodynamics of our curved $\pi$-systems ${ }^{2}$ initiated discussion. Here, we provide our responses with the hope that further indepth discussions will deepen the understanding of curved $\pi$-systems. We also hope that readers will enjoy the discussions on unique supramolecular systems in conjunction with our original paper.

\section{Main conclusion}

The most important finding in our paper is the dual-mode dynamics of the inner fullerene journal, ${ }^{2}$ which successfully explains the peculiar temperature-dependent behavior of the ${ }^{1} \mathrm{H}$ NMR spectrum. ${ }^{3}$ In brief, methylene protons on the shaft moiety appeared as broad signals at $25{ }^{\circ} \mathrm{C}$, sharp signals at $0{ }^{\circ} \mathrm{C}$ and broad signals at $-60{ }^{\circ} \mathrm{C}$ in the VT-NMR spectra. However, the aromatic protons on the outer bearing did not synchronously show this tendency, and the effect of the shaft moiety resulted in a "north" and "south" relationship of the aromatic proton signals throughout this temperature range. The latter observation was ascribed to the single-axis rolling motion of the journal to symmetrize four arylene panels, which successfully explains the persistent presence of the simple north/south

aIST, ERATO, Isobe Degenerate $\pi$-Integration Project and Advanced Institute for Materials Research (AIMR), Tohoku University, Aoba-ku, Sendai 980-8577, Japan. E-mail: isobe@m.tohoku.ac.jp; hirohiko-kono@m.tohoku.ac.jp

${ }^{b}$ Department of Chemistry, Tohoku University, Aoba-ku, Sendai 980-8578, Japan ${ }^{c}$ Graduate School of Systems Engineering, Wakayama University, Sakaedani 930, Wakayama-shi 640-8510, Japan

${ }^{d}$ Institute for Quantum Chemical Exploration, Kaigan 3-9-15, Minato-ku, Tokyo 1080022, Japan relationship. ${ }^{4}$ Our theoretical study ${ }^{2}$ revealed that two different motions, precession and spin, were present in the rolling dynamics. Together with their energy differences (precession $=$ low energy barrier, spin = high energy barrier), these two motions explained the former observation, i.e. the peculiar temperature-dependent behavior of the methylene proton signals. The major conclusion in our study was not questioned or challenged by Cabaleiro-Lago et al. ${ }^{1}$

\section{Brief summary of the theoretical thermodynamics in our paper}

Before we explored the dynamic behavior, we needed to decide on the theoretical methods/models and screen them with several functionals. Believing that the results are informative for other researchers in the field, we presented the data in our paper. $^{2}$

We obtained the theoretical association energies $(\Delta E)$ by comparing the energy of the complex at the static global minimum with the energies of its components. We agree that this procedure is very simple and might be naive (too primitive for theoreticians developing state-of-the-art methods/models but feasible for experimentalists). Fortuitously, we found that the value from the LC-BLYP/6-311G(d) data $(\Delta E=-12.0$ $\left.\mathrm{kcal} \mathrm{mol}^{-1}\right)^{5}$ matched the experimental enthalpy $(\Delta H=-12.5$ $\mathrm{kcal} \mathrm{mol}^{-1}$ ), and using this functional, we revealed the important dual-mode dynamics described above.

We do not deny that this matching $(\Delta E=\Delta H)$ might be merely "fortuitous". ${ }^{1}$ We, however, wished to ask the questions: "How fortuitous is this matching?" and "How general/ubiquitous can such fortuitous matching be with this method?". We believe that these questions are open for further investigation 
and discussion. We thus provided the readers with benchmark results with the hope of seeing more results with this model/ method for other curved $\pi$-systems. Possibly, the LC-BLYP method could be a method of choice for curved $\pi$-systems, especially for those prone to (partial) electron transfer reactions. ${ }^{6,7}$

On the other hand, as discussed in detail below, we do not agree with Cabaleiro-Lago's comment that "the paper is flawed in several crucial aspects of the calculations". ${ }^{1}$ We hope that the readers can also consider fundamental issues in the following discussion for their examination of our paper ${ }^{2}$ or for their own theoretical investigations.

\section{Solvation models}

The first criticism of Cabaleiro-Lago et al. ${ }^{1}$ is of Table 1 in our paper. ${ }^{2}$ Cabaleiro-Lago et al. did not like the use of the polarizable continuum model (PCM) for the solvation treatment. ${ }^{8} \mathrm{We}$ agree with them that the PCM is not perfect. Actually, for the LC-BLYP data, the $\Delta E$ value after the PCM treatment (-9.2 kcal $\left.\mathrm{mol}^{-1}\right)$ was inferior to the in vacuo $\Delta E$ value $(-12.0$ kcal $\mathrm{mol}^{-1}$; see above) in reproducing the experimental $\Delta H$ value in $\mathrm{CH}_{2} \mathrm{Cl}_{2}\left(-12.5 \mathrm{kcal} \mathrm{mol}^{-1}\right.$ ) (the readers should also note that the PCM solvation only resulted in a minor contribution to the association enthalpy). Nonetheless, we decided to incorporate the solvation effects for two reasons: (1) the solvation effects should be considered for dynamics studies and were indeed essential in this case to reproduce and explain the experimental observations (see above) ${ }^{2}$ and (2) the preceding experiments of the association thermodynamics exhibited a considerable solvent dependency (Table 1). ${ }^{3,9}$

Considering that a detailed examination of the solvation model is outside the scope of our study, we did not study this aspect any further. However, if one wishes to investigate theoretical solvation models and discuss their appropriateness, one should look for a model that can reproduce unique solventdependent thermodynamics (Table 1). We also believe that providing a benchmark result using the PCM $\left(\mathrm{CH}_{2} \mathrm{Cl}_{2}\right)$ is helpful for theoreticians wishing to find appropriate solvation models in the future. However, as discussed in detail below, the solvent effects observed in our supramolecular system should be difficult to reproduce by using any simple solvation model. ${ }^{\mathbf{1 0 , 1 1}}$

\section{Problems with the comments - 1: $\Delta H$ or $\Delta G$, that is the question}

The most serious issue with the comments of Cabaleiro-Lago et al. is in their second criticism described from the third paragraph starting with "Also, the procedure for obtaining the final values in solution needs clarification". ${ }^{1}$ We hope that theoreticians or researchers will learn the important bases for the discussion before their examination.

The experimental data used in our comparison were “enthalpy $(\Delta H)$ ", which was clearly described throughout our paper in question ${ }^{2}$ or in other preceding papers. ${ }^{3,9}$ The association enthalpy, $\Delta H$, was directly measured by isothermal titration calorimetry (ITC) (Table 1). As described in ref. 28 of our paper," "ITC analysis directly provides an experimental enthalpy for the association, which enables straightforward comparisons with theoretical energetics without nuisance considerations of entropic terms". Readers who are not familiar with this method may also read a review, for instance the review cited in ref. 27 of our paper in question, ${ }^{2}$ or refer to our preceding papers for the original data., ${ }^{3,9}$ For the convenience of the readers, we quote a few important sentences by Schmidtchen: ${ }^{12}$ "Isothermal titration calorimetry (ITC) offers this opportunity (measuring an exchange of energy and momentum) based on one of the best established fields in physics: thermodynamics. ...free energies are composites of the change in the total number of populated energy levels at the temperature of measurement $T \Delta S^{\circ}$ and the overall change in direct mutual interactions encompassing all participants $\Delta H^{\circ}$. It is the latter quantity that is determined in a time-dependent fashion in an ITC experiment".

Seemingly, Cabaleiro-Lago et al. overlooked the difference between $\Delta H$ and $\Delta G$ and failed to differentiate our approach from Grimme's approach (see below). In this regard, the most important physics are expressed in the equation, " $\Delta G=\Delta H-T \Delta S$ ".

\section{The physics behind the problem}

To clarify the "physics behind the problem", ${ }^{1}$ we briefly summarize the physics that we know from our experiments. The relevant data are shown in Table $1,3,9$ and the additional data from the structure-thermodynamics relationship studies are shown in Table 2. ${ }^{9}$ Although the readers should refer to the original

Table 1 Experimental data for the thermodynamics of $C_{60}$ encapsulation in $(P)-(12,8)-[4] C^{a}$

\begin{tabular}{lcccc}
\hline Solvent & \multicolumn{1}{c}{$\log K_{\mathrm{a}}{ }^{b}$} & $\Delta G^{c}\left(\mathrm{kcal} \mathrm{mol}^{-1}\right)$ & $\Delta H^{d}\left(\mathrm{kcal} \mathrm{mol}^{-1}\right)$ & $\Delta S^{e}\left(\mathrm{cal} \mathrm{mol}^{-1} \mathrm{~K}^{-1}\right)$ \\
\hline 1-Methylnaphthalene & $8.9 \pm 0.0$ & $-12.1 \pm 0.0$ & $-7.4 \pm 0.3$ & $15.9 \pm 1.0$ \\
$o$ DCB & $9.5 \pm 0.2$ & $-13.0 \pm 0.3$ & $-7.7 \pm 0.2$ & $17.6 \pm 1.6$ \\
PhCN & $10.0 \pm 0.2$ & $-13.6 \pm 0.3$ & $-8.0 \pm 0.2$ & $18.9 \pm 1.6$ \\
$\mathrm{PhCl}$ & $10.5 \pm 0.1$ & $-14.3 \pm 0.1$ & $-9.0 \pm 0.2$ & $17.8 \pm 1.1$ \\
$\mathrm{CH}_{2} \mathrm{Cl}_{2}$ & $10.9 \pm 0.0$ & $-14.9 \pm 0.3$ & $-11.6 \pm 0.4$ & $10.9 \pm 1.3$ \\
$\mathrm{CHCl}_{3}$ & $10.9 \pm 0.2$ & $-14.9 \pm 0.3$ & $-11.3 \pm 0.2$ & $15.3 \pm 1.6$ \\
Toluene & $11.6 \pm 0.2$ & $-15.8 \pm 0.3$ & $-13.6 \pm 0.4$ & $15.2 \pm 1.3$ \\
Benzene & $12.2 \pm 0.1$ & $-16.6 \pm 0.1$ & $10.2 \pm 1.8$
\end{tabular}

${ }^{a}$ Data taken from ref. 9. ${ }^{b}$ Measured by fluorescence quenching experiments at $25{ }^{\circ} \mathrm{C} .{ }^{c}$ Obtained from the corresponding $K_{\mathrm{a}}$ value. ${ }^{d}$ Directly measured by ITC experiments. ${ }^{e}$ Obtained from the corresponding $\Delta G$ and $\Delta H$ values at $25^{\circ} \mathrm{C}$. 
Table 2 Experimental data for the thermodynamics of fullerene encapsulation in oDCB ${ }^{a}$

\begin{tabular}{lllll}
\hline Complex & $\log K_{\mathrm{a}}{ }^{b}$ & $\Delta G^{c}\left(\mathrm{kcal} \mathrm{mol}^{-1}\right)$ & $\Delta H^{d}\left(\mathrm{kcal} \mathrm{mol}^{-1}\right)$ & $\Delta S^{e}\left(\mathrm{cal} \mathrm{mol}^{-1} \mathrm{~K}^{-1}\right)$ \\
\hline$(P)-(12,8)-[4] \mathrm{CC} \supset \mathrm{C}_{60}$ & $9.5 \pm 0.2$ & $-13.0 \pm 0.3$ & $-7.7 \pm 0.2$ & $17.6 \pm 1.6$ \\
$(P)-(12,8)-[4] \mathrm{CC} \supset \mathrm{C}_{70}$ & $9.6 \pm 0.1$ & $-13.1 \pm 0.1$ & $-10.1 \pm 0.2$ & $10.0 \pm 1.1$ \\
$(P)-(12,8)-[4] \mathrm{CA} \supset \mathrm{C}_{60}$ & $9.7 \pm 0.1$ & $-13.2 \pm 0.1$ & $-13.7 \pm 0.3$ & $-1.5 \pm 1.5$ \\
$(P)-(12,8)-[4] \mathrm{CA} \supset \mathrm{C}_{70}$ & $9.6 \pm 0.3$ & $-13.1 \pm 0.4$ & $-14.3 \pm 0.1$ & $-4.1 \pm 1.7$
\end{tabular}

${ }^{a}$ Data taken from ref. 9. ${ }^{b}$ Measured by fluorescence quenching experiments at $25{ }^{\circ} \mathrm{C} .{ }^{c}$ Obtained from the corresponding $K_{\mathrm{a}}$ value. ${ }^{d}$ Directly measured by ITC experiments. ${ }^{e}$ Obtained from the corresponding $\Delta G$ and $\Delta H$ values at $25^{\circ} \mathrm{C}$.

papers for each conclusion, the data discloses important experimental facts that should be considered in theoretical calculations.

\section{Enthalpy}

The enthalpy $(\Delta H)$ has an associative contribution $(\Delta H<0)$ in $\Delta G$. The $\Delta H$ value depends on the $\mathrm{C}-\mathrm{C}$ contact areas (Table 2), ${ }^{9}$ and van der Waals (vdW) interactions play the dominant role in $\Delta H$.

\section{Entropy}

The entropy $(\Delta S)$ also has an associative contribution $(\Delta S>0$; $-T \Delta S<0)$ in the $\Delta G$ of [4]CC complexes. We concluded that a positive $\Delta S$ with [4]CC originated mainly from the desolvation upon complexation (Table 1)..$^{\mathbf{1 3 , 1 4}}$ In addition, contributions from alkyl conformations should not be neglected in $\Delta S$. Entropy contributions from alkyl conformations resulted in an opposite, dissociative entropy $(\Delta S<0 ;-T \Delta S>0)$ in $\Delta G$ with a [4]CA congener possessing restricted alkyl conformations (Table 2). ${ }^{9}$ Thus, both solvation and alkyl conformations must be considered to understand or reproduce $\Delta S$.

\section{Our approach: $\Delta H$}

The modern analysis using ITC provides direct experimental data of the association enthalpy $(\Delta H)$ (see above). Comparing the experimental $\Delta H$ value with the theoretical $\Delta E$ value, we were able to avoid complicated issues dealing with the entropy terms. ${ }^{15,16}$ The experimental enthalpy provided new reference data for theoreticians to examine their theoretical methods/ models. Thus, the following question should be addressed: "What are the suitable theoretical models/methods to reproduce (and understand) the experimental enthalpy?"17

This approach of ours ( $\Delta H v s . \Delta E$ ) is different from that of Grimme's ( $\Delta G$ vs. $\Delta G_{\text {theo }}$ ). Using the experimental enthalpy, we can avoid a theoretical calculation that is "quite costly and poses some problems related to low-frequency modes (sic)"1 (see below for more serious issues). We do not ignore the "physics behind the problem"1 but, rather, we respect the physics that we know from the experiments.

\section{Problems with the comments $-2: \Delta G$}

Cabaleiro-Lago et al. provided their preliminary theoretical results adopting Grimme's approach $\left(\Delta G v s . \Delta G_{\text {theo }}\right)$. The issues involved in Cabaleiro-Lago's treatment may also be instructive for the readers in understanding the fundamental physics.

Grimme's unique and elaborate approach using the rigidrotor harmonic oscillator (RRHO) term was developed for the theoretical prediction of the Gibbs free energies for the association. ${ }^{18,19}$ This approach $\left(\Delta G v s . \Delta G_{\text {theo }}\right)$ is also reasonable for the comparison of various experimental data sets because of a scarcity of experimental $\Delta H$ values. Most (classic) experiments on supramolecular complexes provided only the $K_{\mathrm{a}}$ values or the $\Delta G$ values. Grimme's approach indeed successfully reproduced several experimental $\Delta G$ values with $\Delta G_{\text {theo, especially in }}$ combination with dispersion-corrected functionals. We do not deny this important fact. However, this approach is not free from serious errors when it is incorrectly applied.

The issue with Cabaleiro-Lago's results ${ }^{1}$ originates from the entropy. Grimme clearly stated that his approach is applicable "if the other contributions (solvation and entropy change) could be obtained within similar error limit"18 (1-2 $\left.\mathrm{kcal} \mathrm{mol}^{-1}\right)$. Our supramolecular system is a difficult case that should not be treated with Grimme's approach without in-depth consideration of the physics behind the problem: the experimental entropy $(\Delta S)$ involves considerable effects of solvation and conformations (see above).,

Experimentally, the entropy contribution $(-T \Delta S)$ in the $\Delta G$ of our system is a negative value $\left(-3.3 \mathrm{kcal} \mathrm{mol}^{-1}\right.$ in $\mathrm{CH}_{2} \mathrm{Cl}_{2}$, largest with $-5.6 \mathrm{kcal} \mathrm{mol}^{-1}$ in $\mathrm{PhCN}$ at $25{ }^{\circ} \mathrm{C}$; see Table 1). Qualitatively, this entropy contribution should be incorporated in the RRHO term (see eqn (1) of ref. 18 for the correct, original equation) in Grimme's approach. However, the RRHO terms (or even the $\Delta G_{\text {solv }}$ terms) in Cabaleiro-Lago's results are large positive values, and we are unsure if these values are correctly reflecting the real physics. (Could Cabaleiro-Lago's results draw a correct picture where our supramolecular bearing is assembled favorably by both enthalpy and entropy terms?) In addition, because the alkyl conformation is another important contributor to the $\Delta S$ (see above), the long hexyl chains should not be modeled using simplified methyl groups in their $\Delta G_{\text {theo }}$ analysis. The $\Delta G_{\text {theo }}$ analysis without the proper entropy considerations is worse than "walking on incredibly thin ice" 1 and ignores "one of the best established fields in physics". ${ }^{12}$

The data presented by Cabaleiro-Lago et al. may be interesting as a preliminary starting point. For further in-depth investigations, they should consider the above discussion with special consideration for the entropy term. ${ }^{\mathbf{2 0 , 2 1}}$ The readers should also note that Cabaleiro-Lago's discussion regarding the 
solvation models requires further extensive investigations to come to a conclusion (the solvation model that reproduces the experimentally observed "solvent dependency" should be located). Until they can provide thorough discussion and supporting data, their data merely demonstrate a "fortuitous agreement of $\Delta G_{\text {theo }}=\Delta G$ " without reflecting the physics. ${ }^{22}$

\section{Outlook}

We fully agree with Cabaleiro-Lago et al. that there is much to debate and investigate in the thermodynamics of our supramolecular systems. Although the system may be challenging for theoretical investigations in various aspects, we hope that our papers and this response may stimulate many more theoreticians to join the investigations and discussion (as described above, sufficient experimental data have illustrated the physics for the association). Hopefully, from high-quality theoretical investigations, we can better understand the unique chemistry and physics of curved $\pi$-systems.

\section{References}

1 E. M. Cabaleiro-Lago, J. Rodriguez-Otero and A. Gil, Chem. Sci., 2016, 7, DOI: 10.1039/C5SC04676A.

2 H. Isobe, K. Nakamura, S. Hitosugi, S. Sato, H. Tokoyama, H. Yamakado, K. Ohno and H. Kono, Chem. Sci., 2015, 6, 2746-2753.

3 H. Isobe, S. Hitosugi, T. Yamasaki and R. Iizuka, Chem. Sci., 2013, 4, 1293-1297.

4 S. Sato, T. Yamasaki and H. Isobe, Rolling dynamics was also confirmed using solid-state NMR analyses, Proc. Natl. Acad. Sci. U. S. A., 2014, 111, 8374-8379.

5 H. Iikura, T. Tsuneda, T. Yanai and K. Hirao, J. Chem. Phys., 2001, 115, 3540-3544.

6 S. Hitosugi, K. Ohkubo, Y. Kawashima, T. Matsuno, S. Kamata, K. Nakamura, H. Kono, S. Sato, S. Fukuzumi and H. Isobe, Chem.-Asian J., 2015, 10, 2404-2410; S. Hitosugi, K. Ohkubo, R. Iizuka, Y. Kawashima, K. Nakamura, S. Sato, H. Kono, S. Fukuzumi and H. Isobe, Org. Lett., 2014, 16, 3352-3355.

7 See our original paper in ref. 2 for more references.

8 J. Tomasi, B. Mennucci and R. Cammi, Chem. Rev., 2005, 105, 2999-3093.

9 T. Matsuno, S. Sato, R. Iizuka and H. Isobe, Chem. Sci., 2015, 6, 909-916; S. Hitosugi, R. Iizuka, T. Yamasaki, R. Zhang, Y. Murata and H. Isobe, Org. Lett., 2013, 15, 3199-3201.

10 Y. Marcus, A. L. Smith, M. V. Korobov, A. L. Mirakyan, N. V. Avramenko and E. B. Stukalin, J. Phys. Chem. B, 2001,
105, 2499-2506; Y. Marcus, J. Phys. Chem. B, 1997, 101, 8617-8623.

11 For instance, see: R. Ludwig and A. Appelhagen, Angew. Chem., Int. Ed., 2005, 44, 811-815; H. Isobe, T. Homma and E. Nakamura, Proc. Natl. Acad. Sci. U. S. A., 2007, 104, 14895-14898.

12 F. P. Schmidtchen, in Supramolecular Chemistry: From Molecules to Nanomaterials, ed. J. W. Steed and P. A. Gale, Wiley, Chichester, 2012, vol. 2, pp. 275-296.

13 In solution, crystalline solvates of fullerene form (ref. 10), which may also be common in many other curved $\pi$-systems.

14 The positive $\Delta S$ may also support an anomalous, interesting feature of our system: the fullerene molecule maintains, at least to some extent, rotational degrees of freedom even in the bearing.

15 In this regard, Cabaleiro-Lago's statement "the apparent success of $L C-B L Y P$ relies on the cancelation of the contributions coming from the RRHO term, dispersion and the description of solvent effects (ref. 1)" is correct if we read "RRHO term, dispersion and the description of solvent effects" as "-T $S$ ".

16 In passing, the enthalpy-entropy compensation should be discussed with care in the theoretical studies, A. CornishBowden, J. Biosci., 2002, 27, 121-126; D. M. Ford, J. Am. Chem. Soc., 2005, 127, 16167-16170.

17 We do not think that our primitive calculation model is the best solution: this question is widely open for further investigations.

18 S. Grimme, Chem.-Eur. J., 2012, 18, 9955-9964.

19 J. H. Jensen, Phys. Chem. Chem. Phys., 2015, 17, 12441-12451.

20 They may also wish to incorporate the symmetry effects on rigid-rotor rotational entropy (see ref. 19). Our outer bearing is in the $D_{4}$ symmetry with $(P)$-helicity. The inner journals are in the $C_{2 \mathrm{v}}$ symmetry for the shafted journal, the $C_{\mathrm{s}}$ symmetry for the shafted journal in the protonated form and the $I_{\mathrm{h}}$ symmetry for the $\mathrm{C}_{60}$ journal.

21 Readers whoare interested in the results of the dispersioncorrected functionals in our approach should refer to Table S1 in our paper (ref. 2). These data as well as Cabaleiro-Lago's best results $\left(\Delta E_{\text {comp }}=-82.05 \mathrm{kcal} \mathrm{mol}^{-1}\right.$; B3LYP-D3) indicated that our non-covalent assembly could rival covalent bonds in the association energies. For instance, the $\mathrm{C}-\mathrm{H}$ bond dissociation energy (enthalpy) of cyclopentadiene is $71.1 \mathrm{kcal} \mathrm{mol}^{-1}$. See D. F. McMillen and D. M. Golden, Annu. Rev. Phys. Chem., 1982, 33, 493-532.

22 Cabaleiro-Lago et al. incorrectly compared the experimental $\Delta G$ in $o$ DCB with their $\Delta G_{\text {theo }}$ in $\mathrm{CH}_{2} \mathrm{Cl}_{2}$ (ref. 1). 